\title{
Evaluation of pericardiocentesis videos on YouTube as a reliable source for on-line education in the COVID-19 period
}

\author{
Suleyman Cagan Efe ${ }^{1}$, Sedat Kalkan², Ali Karagoz ${ }^{1}$ \\ ${ }^{1}$ Department of Cardiology, Kartal Kosuyolu Heart and Research Hospital, Istanbul, Turkey \\ ${ }^{2}$ Departemnt of Cardiology, Pendik State Hospital, Istanbul, Turkey
}

Kardiochir Torakochir Pol 2021; 18 (1): 33-39

\begin{abstract}
Introduction: Pericardiocentesis is an invasive procedure performed to drain fluid from the pericardial cavity.

Aim: We investigated the quality of videos about pericardiocentesis on YouTube and analysed their reliability and teaching properties, in the covid 19 period when online education has come to the fore.

Material and methods: We searched YouTube using the terms „pericardiocentesis, cardiac tamponade, pericardial effusion, pericardial effusion drainage, pericardial tamponade” for uploaded videos. We scored every video according to the questions we prepared using the guidelines about pericardiocentesis. We used the HONcode score, GQS score, and RELIABILITY Score to assess the quality of videos. Two physicians independently and blindly classified videos as useful or misleading and rated them. Results: A total of 168 videos were examined. After the application of exclusion criteria, 38 videos were evaluated. The pericardiocentesis checklist average score was $10.45 \pm 2.56$. According to sources of videos, the average score for university or research hospital videos was $13.1 \pm 1.5$, and videos whose source could not be identified had an average score of 7.5 \pm 2.0 . According to the level of HONcode, 17 (44.7\%) videos were low quality; according to GQS score, 8 (21.1\%) videos were poor quality. The quality of university hospital uploads ( $\beta$-coefficient 3.960, $p$-value 0.004 ) were higher and statistically significant than other upload centres.

Conclusions: The educational value of pericardiocentesis videos on YouTube are low. It is recommended that doctors and patients be aware of and adopt the developing technology, and they should prefer videos uploaded from university hospitals and educational hospitals.
\end{abstract}

Key words: pericardiocentesis, cardiac tamponade, YouTube, COVID-19.

\section{Introduction}

Accumulation of fluid in the pericardial cavity is a lifethreatening condition. Fluid collection may be due to cardiac or non-cardiac causes, but the results can be dramatic. Drainage of the fluid accumulated in the pericardial cavity is called pericardiocentesis and is performed by cardiologists and emergency medicine professionals as an acute therapeutic procedure for life-threatening conditions. It was first described in 1653 by Riolanus. The technique and application of pericardiocentesis have evolved over the years. As stated in the ESC position paper related to pericardiocentesis, procedures can be performed from different approaches (subxiphoid, apical, and parasternal) and with different guides (echocardiography guided, fluoroscopyguided, and electrocardiography guided) [1, 2]. Although pericardiocentesis is performed in the light of theoretical information, it is beneficial for the clinician to see how the procedure is performed.

Almost 4.54 billion people were active internet users as of January 2020, encompassing $59 \%$ of the global population [3]. Today, the internet and social media have played a part in the lives of people from all social layers of life. The probability of disseminating misleading information to healthcare consumers is high and can have catastrophic implications [4]. Video sharing channels, which are accessible to everyone, provide visual information to viewers. YouTube is the largest video sharing site, with over 300 hours of video uploaded every minute [5]. Unlike journal articles or textbooks that are heavily reviewed, there is no review process for the videos being uploaded on YouTube. For this reason, research to establish the accuracy and reliability of these educational videos is crucial.

Address for correspondence: Suleyman Cagan Efe MD, Department of Cardiology, Kartal Kosuyolu Heart and Research Hospital, Istanbul, Turkey, phone: +90 2165001500, e-mail: scaganefe@gmail.com

Received: 12.10 .2020 , accepted: 6.01.2021. 
There are some tools that were created for measuring the quality of videos or health-associated internet sites; the DISCERN score, HONcode score, GQS score, RELIABILITY score, and JAMA benchmark instrument are the most used ones of them [6-9].

\section{Aim}

In our study, we tried to determine the medical suitability of videos related to pericardiocentesis, which were shared on YouTube, which is easily accessible, free, and popular. Their reliability was determined by using evaluation methods that were suitable for our study, such as HONcode score, GQS score, and RELIABILITY score. We aimed to test and characterize the content and quality of information on pericardiocentesis in YouTube videos in the COVID-19 pandemic period.

\section{Material and methods}

We conducted this study to identify the educational properties of pericardiocentesis procedure videos on YouTube. The YouTube (www.YouTube.com) search bar was checked using the keywords 'pericardiocentesis', 'cardiac tamponade', 'pericardial effusion', 'pericardial effusion drainage', and 'pericardial tamponade'. The collection of all videos containing information regarding pericardiocentesis was conducted on 10 March 2020. We did not use any personal Google or YouTube account for the video search. The search yielded 168 videos in total; after applying exclusion criteria, there were 38 remaining videos to examine. Videos were ranked by relevance and screened for information on

Table I. Pericardiocentesis checklist according to consensus reports

\begin{tabular}{|c|c|}
\hline Question & Rating \\
\hline 1. Is the patient's position suitable? & 01 \\
\hline 2. Is there a saturation probe? & 01 \\
\hline 3. Is the patient's arterial blood pressure monitored? & 01 \\
\hline 4. Does the patient have a central venous catheter? & 01 \\
\hline $\begin{array}{l}\text { 5. Does the patient have electrocardiographic } \\
\text { monitorization? }\end{array}$ & 01 \\
\hline 6. Was appropriate sterilization performed? & 01 \\
\hline $\begin{array}{l}\text { 7. Was a guiding instrument (echocardiography, } \\
\text { fluoroscopy, electrocardiography) used during the } \\
\text { process? }\end{array}$ & 01 \\
\hline 8. Is there anyone who assists in the process? & 01 \\
\hline 9. Is sedation used? & 01 \\
\hline 10. Is the needle entry angle appropriate? & 01 \\
\hline 11. Is needle insertion suitable? & 01 \\
\hline 12. Is the needle advanced by aspiration? & 01 \\
\hline 13. Is the coagulation of aspirate fluid checked? & 01 \\
\hline $\begin{array}{l}\text { 14. Was the needle was confirmed to be in the pericardial } \\
\text { cavity? }\end{array}$ & 01 \\
\hline 15. Was a sheet placed accordingly guideline and closed? & 01 \\
\hline
\end{tabular}

pericardiocentesis. We collected the following quantitative data: view counts, comments, likes, dislikes, and duration. All videos were viewed and analysed by 2 independent physicians who have sufficient experience in pericardiocentesis procedures (SCE, SK); in the event of a discrepancy, a third reviewer arbitrated the disagreement. Videos were rated by researchers according to HONcode score, GQS score, and RELIABILITY score.

\section{Inclusion and exclusion criteria}

- Videos not using English language were not included in the study.

- Only one video was considered if the same video was repeated or if the video was found in parts.

- Videos with incompatible content were not included in the study.

- Animated videos were not included in the study.

- Videos shorter than 30 seconds were not included in the study.

\section{Each video was categorized into 4 centres according to the upload source}

1. Patient or miscellaneous (when identifying data could not be found or determined).

2. Physician.

3. Educational channel.

4. University or Educational Hospital.

\section{Pericardiocentesis Checklist}

We prepared a checklist according to pericardiocentesis consensus papers [2, 10, 11]. We prepared 15 questions. We rated the videos according to the questions (no: 0 , yes: 1 ) in this checklist. Videos with a rating of more than $80 \%$ of questions were defined as good quality videos (Table I).

\section{Reliability of information score}

All videos were analysed with a RELIABILITY score in terms of the reliability and integrity of their information based on a 5-point scale. The RELIABILITY score, which is derived from the DISCERN score, attempts to determine the reliability of the information based on 5 questions [7]. The result can reach a maximum of 5 points by giving 1 point for each question covered in the video. The reliability of information questions can be seen in Table II.

Table II. Reliability of information questions

\begin{tabular}{lc} 
Question & Rating \\
1. Are the aims clear and achieved in the video? & 01 \\
\hline 2. Are reliable sources of information used in the video? & 01 \\
\hline $\begin{array}{l}\text { 3. Is the information presented balanced and } \\
\text { unbiased in the video? }\end{array}$ & 01 \\
\hline $\begin{array}{l}\text { 4. Are additional sources of information listed for } \\
\text { patient reference? }\end{array}$ & 01 \\
\hline \begin{tabular}{l} 
5. Are areas of uncertainty mentioned in the video? \\
\hline
\end{tabular} & 01 \\
\hline
\end{tabular}


Table III. GQS description questions

\begin{tabular}{lc} 
Question & Rating \\
$\begin{array}{l}\text { 1. Poor quality, poor flow of the video, most } \\
\text { information missing, not at all useful for patients }\end{array}$ & 01 \\
\hline $\begin{array}{l}\text { 2. Generally poor quality and poor flow, some } \\
\text { information listed but many important topics }\end{array}$ & 01 \\
missing, of very limited use to patients & 01 \\
\hline $\begin{array}{l}\text { 3. Moderate quality, suboptimal flow, some important } \\
\text { information is adequately discussed but others }\end{array}$ & 01 \\
\hline poorly discussed, somewhat useful for patients & \\
\hline $\begin{array}{l}\text { 4. Good quality and generally good flow. Most of the } \\
\text { relevant information is listed, but some topics not } \\
\text { covered, useful for patients }\end{array}$ & 01 \\
\hline \begin{tabular}{l} 
5. Excellent quality and flow, very useful for patients \\
\hline
\end{tabular}
\end{tabular}

\section{GQS description}

All videos were also rated using a global quality score (GQS) that uses a 5-point scale to rate the overall quality of the video. The GQS score is an assessment to ensure that the quality of information and the reviewer decide how useful a particular video will be for a patient [8]. The GQS questions can be seen in Table III.

\section{HONcode Score}

The HONcode is one of the oldest methods used to present and evaluate health-related information on the internet. Studies have shown that websites that comply with the HONcode principles provide quality health information to patients and healthcare professionals. The HONcode tool, consisting of 8 adapted questions, is used to evaluate the quality of the videos. For each question, the evaluator states whether the principle is followed in the video (1: yes, $0:$ no), and the video is evaluated by calculating the total HONcode score [6].

The HONcode adherence level was determined by total score: 0-2 means low adherence; 3-5 means medium adherence; and 6-8 means high adherence. HONcode questions can be seen in Table IV.

\section{Ethics}

Because of the anonymity of the data, there was no need to obtain ethical approval.

\section{Statistical analysis}

For statistical analysis, R software version 4.02 (Vienna, Austria), 'Hmisc', 'rms' packed was used. Describing the continuous data, we used mean \pm standard deviation (for normal distribution), and the median and interquartile range (25-75 IQR) for non-normal distribution. Frequency and percentage were given for categorical data, and the $\chi^{2}$ test was used to compare these parameters. The Shapiro-Wilk test and histogram graphic analysis were used to check the normality of data for quantitative variables.

A correlation test was applied for HONcode, GQS, RELIABILTY scores, and the pericardiocentesis checklist score. The
Table IV. HONcode instrument questions

\begin{tabular}{lc} 
Question & Rating \\
1. Any medical or health advice given in the video & 01 \\
must come from a qualified health professional & \\
unless it is clearly stated that the information does & \\
not come from a qualified health source & \\
\hline 2. The information provided in the videos must be & 01 \\
designed to support the patient's self-management & \\
but is not meant to replace a patient-physician & \\
relationship & \\
\hline 3. The information in the video maintains the right to & 01 \\
confidentiality and respect of the individual patient & \\
featured & \\
\hline 4. Each video contains references to source data on & 01 \\
information presented or contains a specific HTML & \\
link to source information & \\
\hline 5. Each video containing claims on the benefits or per- & 01 \\
formance of specific, skills/behaviours, interventions, & \\
treatments, products, and so on must be supported & \\
by evidence through references or HTML links & \\
\hline 6. The video must provide the viewer with contact & 01 \\
information, or contain a website link to more \\
information
\end{tabular}

Pearson test was applied if data had a normal distribution, and if not, the Spearman correlation test was applied. The Cronbach- $\alpha$ value was evaluated for inter-rater assessments such as HONcode, GQS, and total pericardiocentesis checklist score. Among 4 upload centre group scores of HONcode, pericardiocentesis checklist, GQS, and RELIABILTY instruments were tested with one-way ANOVA or Kruskal-Wallis (non-normal distribution) for numerical variables; if needed, sub-group analysis with Tukey post hoc test was applied.

With linear regression, the most informative parameter pericardial checklist was taken as a dependent variable to the model as numeric. Independent variables were as follows: HONcode, GQS, likes, dislikes, and video duration. The statistical significance was considered as $p$-value $<0.05$.

\section{Results}

A total of 168 videos were examined. After the application of the exclusion criteria, 38 videos were evaluated. A sample size of 38 meets the guidelines for conducting reliability analyses in media content analyses (i.e. at least 50 cases or 10 to $20 \%$ of the sample). When looking at the video sources, it was determined that $8(21.1 \%)$ videos originated from a university or research hospital, 6 (15.8\%) 
videos originated from educational sites, 18 (47.4\%) videos were shared by individual health workers, and 6 (15.8\%) videos could not be identified. The average duration of the videos was $2 \pm 0.6$ minutes, and the average number of views was $16.799 \pm 4890$. The average number of likes was $43 \pm 12$, and the average number of dislikes was $4 \pm 1$. The number of average comments was $2 \pm 0.7$ (Table V).

Cronbach's $\alpha$ method was used for interrater reliability analysis. Values for HONcode score and total pericardiocentesis score were found to be 0.917 and 0.953 , respectively.

\section{Pericardiocentesis checklist}

According to the checklist, which was prepared according to the pericardiocentesis consensus reports, the average score was $10.45 \pm 2.56$. According to sources of videos, the average score of university or research hospital videos was $13.1 \pm 1.5$, the average score of educational site videos was $12 \pm 1.4$, individual health workers videos averaged $9.7 \pm 1.9$, and videos that could not be identified averaged 7.5 \pm 2.0 (Table IV).

According to the checklist, questions 11 ( $n=37,97.4 \%)$ and 9 ( $n=35,92.1 \%)$ were the hughest rated ones; on the other hand, questions $4(n=10,26.3 \%)$ and $14(n=13$, $34.2 \%)$ were the least rated ones.

\section{Checklist questions and their ratings (n: number of videos, \% percentage)}

Is the patient's position suitable? $(n=28,73.7 \%)$

Is there a saturation probe? $(n=21,55.3 \%)$
Is the patient's arterial blood pressure monitored? $(n=30,78.9 \%)$

Does the patient have a central venous catheter? $(n=10,26.3 \%)$

Does the patient have electrocardiographic monitoring? $(n=31,81.6 \%)$

Has appropriate sterilization been performed? $(n=31$, $81.6 \%)$

Was a guiding instrument (echocardiography, fluoroscopy) used during the process? ( $n=26,68.4 \%)$

Did anyone assist in the process? $(n=25,65.8 \%)$

Was sedation used? ( $n=35,92.1 \%)$

Was the needle entry angle appropriate? $(n=32,84.2 \%)$

Was the needle insertion suitable? ( $n=37,97.4 \%)$

Was the needle advanced by aspiration? ( $n=34,89.5 \%)$

Was the coagulation of aspirate fluid checked? $(n=13$, $34.2 \%)$

Was the needle confirmed to be in the pericardial cavity? $(n=16,42.1 \%)$

Was a sheet placed accordingly and closed? $(n=28,73.7 \%)$

\section{HONcode}

A majority of the videos met the first question criterion, which required medical and health advice to come from a qualified health professional, unless clearly stated otherwise ( $n=34,89.5 \%$ ). Videos meeting the criterion for the second question, which was providing patient education on pericardiocentesis self-management without the intention to replace the patient-physician relationship, was more

Table V. Video parameters and scores

\begin{tabular}{|c|c|c|c|c|c|c|}
\hline Parameter & 1 & 2 & 3 & 4 & All groups & $P$-value \\
\hline Number of videos, $n(\%)$ & $6(15.8)$ & $18(47.4)$ & $6(15.8)$ & $8(21.1)$ & & \\
\hline Duration [min] & & & & & $2 \pm 0.6$ & \\
\hline View & & & & & $16.799 \pm 4890$ & \\
\hline Like & & & & & $43 \pm 12$ & \\
\hline Dislike & & & & & $4 \pm 1$ & \\
\hline Comment (number) & & & & & $2 \pm 0.7$ & \\
\hline \multicolumn{7}{|c|}{ Pericardiocentesis Checklist Score: } \\
\hline Score (given as mean) & $7.5 \pm 2.0$ & $9.7 \pm 1.9$ & $12 \pm 1.4$ & $13.1 \pm 1.5$ & $10.45 \pm 2.56$ & $<0.001$ \\
\hline $95 \%$ confidence interval & $(5.32,9.68)$ & $(8.78,10.67)$ & $(10.52,13.48)$ & $(11.83,14.42)$ & & \\
\hline \multicolumn{7}{|l|}{ GQS Score: } \\
\hline Mean & $1.33 \pm 0.51$ & $2.17 \pm 0.78$ & $3.5 \pm 0.83$ & $3.38 \pm 1.30$ & $2.5 \pm 1.15$ & $<0.001$ \\
\hline $95 \%$ confidence interval & $(0,79,1.88)$ & $(1.78,2.56)$ & $(2.62,4.38)$ & $(2.29,4.46)$ & $(2.12,2.88)$ & \\
\hline \multicolumn{7}{|l|}{ HONcode: } \\
\hline Mean & $1.17 \pm 0.98$ & $2.56 \pm 1.46$ & $4.17 \pm 1.94$ & $4.63 \pm 2.13$ & $3.03 \pm 1.97$ & 0.001 \\
\hline $95 \%$ confidence interval & $(0.13,2.20)$ & $(1.83,3.28)$ & $(2.13,6.20)$ & $(2.84,6.41)$ & $(2.38,3.68)$ & \\
\hline \multicolumn{7}{|l|}{ RELIABILITY: } \\
\hline Mean & $2.0 \pm 0$ & $2.44 \pm 0.85$ & $3.33 \pm 0.81$ & $3.13 \pm 0.99$ & $2.66 \pm 0.90$ & 0.016 \\
\hline $95 \%$ confidence interval & $(2.0,2.0)$ & $(2.02,2.87)$ & $(2.48,4.19)$ & $(2.30,3.95)$ & $(2.36,2.96)$ & \\
\hline
\end{tabular}


than half ( $n=25,65.8 \%$ ). Also, nearly half of the videos maintained the right to confidentiality and respect of patients, as featured in the third question ( $n=18,47.4 \%$ ). The number of videos meeting the criterion for the fourth question, which contained references to source data on the information presented, was low ( $n=11,28.9 \%)$. The number of videos meeting the fifth criterion, which was requiring claims to be supported by evidence through references or hypertext markup language (HTML) links, was low $(n=7$, $18.4 \%)$. For the sixth criterion, which asked about additional contact information and/or a website link for further information, was met by one-third of the videos $(n=13$, $34.2 \%$ ). The 2 HONcode questions with the fewest videos in compliance were the seventh question, which identified funders and contributors of information presented in the video or within video descriptions ( $n=3,7.9 \%)$ and the eighth question, which only applied to videos supported by funding from advertisers ( $n=5,13.2 \%)$.

The mean HONcode score was $3.12 \pm 1.95$ and $2.91 \pm 1.99$ between raters, respectively, and the mean score was 3.03 \pm 1.97 (Table V).

\section{RELIABILITY score}

The reliability of the information questionnaire score was $2.62 \pm 1.12$ and $2.70 \pm 0.68$ between raters, respectively, and the average score was $2.66 \pm 0.90$ (Table V).

\section{GQS Score}

The GQS description score was $2.56 \pm 1.12$ and 2.44 \pm 0.18 between raters, respectively, and the mean score was $2.5 \pm 1.15$ (Table V).

\section{Quality of videos}

According to the pericardiocentesis checklist score, the number of videos that responded positively to more than $80 \%$ of the questions was 8 (21). Only $21 \%$ of the videos were evaluated as compatible with the position paper of ESC according to the level of HONcode adherence (low, medium, and high). The majority of videos across all media categories were rated as low quality ( $n=17,44.7 \%)$, 16 (42.1\%) were judged to be medium quality, and 5 (13.2) and were judged to be high quality.

According to the GQS score, video qualities were as follows: 8 (21.1\%) were poor quality, 13 (34.2\%) were generally poor quality and poor flow videos, 9 (23.7\%) were moderate quality, 6 (15.8\%) were good quality and generally good flow videos, and 2 (5.3\%) videos were excellent quality (Table VI).

Pericardiocentesis checklist scores of videos and changes according to HONcode, RELIABILTY, and video duration are shown in gg plots in Figure 1. The graph shows that the parameters are increasing linearly.

It was observed that there was a statistical difference between GQS Score, HONCODE, and RELIABILTY scores of the videos that were found to be appropriate or not appropriate according to the pericardiocentesis checklist. Mean scores of videos according to pericardiocentesis checklist quality can be seen in Table VII.

Upload centre 1 was defined as a reference for comparing other upload centres. Total pericardiocentesis checklist score, which is the main parameter in terms of teaching and training features of the videos was checked according to videos score and parameters. The parameters predicting the total pericardiocentesis checklist score was analysed in a multivariable linear regression model. The university hospital uploads ( $\beta$-coefficient 3.960, $p$-value 0.004) were statistically more significant than other upload centres, but other parameters (such as HONcode, reliability, video duration, like, dislike) included in the model were not statistically significant (Table VIII).

\section{Discussion}

Although invasive procedures can be learned theoretically, the value of visual sources cannot be ignored in terms of consolidating information. In our study, we tried to determine the accuracy of tutorials in the videos shared on YouTube regarding pericardiocentesis attempts. According to the checklist, which was based on the consensus papers of ESC with a maximum score of 15 , we found that only $8(21 \%)$ of these videos met the criteria with sufficient information by maintaining a score of more than 12 . We determined that $5(13.2 \%)$ of the videos were of suitable quality according to the HONcode score. In the evaluation we made in light of these results, we determined that the quality of videos shared on YouTube about pericardiocentesis education is low. We have shown that these videos may not be suitable for educational purposes.

YouTube is a public portal that creates a platform for everyone to share. This may have beneficial aspects as well as misleading ones. YouTube videos contain great heterogeneity in terms of image quality, primary upload purpose, upload source, and reliability. As we have seen during the COVID-19 pandemic, when online education came to the fore, the reliability of videos uploaded to video sites is of great importance. However, there is no evaluation process before uploading these videos, and everyone can share. It has been shown that the number of views and the amount of likes of the videos are not significant in terms of their reliability [12]. In our study, it was seen that the video-sharing made especially by university hospitals or training hospitals was statistically high compared to individual posts. For this reason, our opinion for healthcare professionals and pa-

Table VI. Quality of videos

\begin{tabular}{lccc} 
According to & Poor quality & Good quality & $\begin{array}{c}\text { Excellent } \\
\text { quality }\end{array}$ \\
$\begin{array}{l}\text { Pericardiocentesis } \\
\text { Checklist Score }\end{array}$ & $30(79 \%)$ & $8(21 \%)$ & \\
\hline GQS Score & $30(78.8 \%)$ & $8(21.2 \%)$ & \\
\hline HONcode & $17(44.7 \%)$ & $16(42.1 \%)$ & $5(13.2 \%)$ \\
\hline RELIABILITY & $31(81.6 \%)$ & $7(18.4 \%)$ & \\
\hline
\end{tabular}



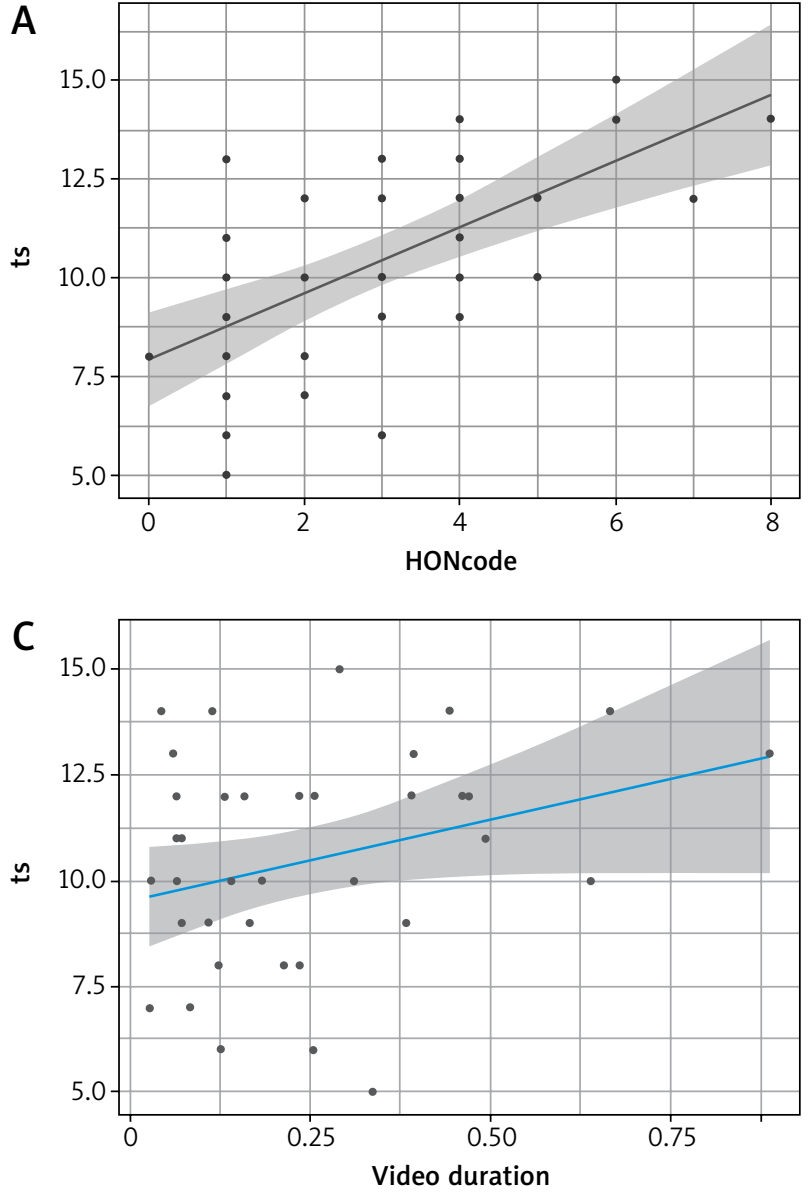

Table VII. Mean scores of videos according to Pericardiocentesis Checklist Scores

\begin{tabular}{lccc} 
Parameter & $\begin{array}{c}\text { Pericardiocentesis } \\
\text { checklist score } \\
\text { good or excellent } \\
(n=8,21 \%)\end{array}$ & $\begin{array}{c}\text { Pericardiocentesis } \\
\text { checklist score poor } \\
(n=30,79 \%)\end{array}$ & P-value \\
GQS Score & $4.0 \pm 0.81$ & $2.16 \pm 0.93$ & $<0.001$ \\
\hline HONcode & $5.29 \pm 1.7$ & $2.52 \pm 1.67$ & $<0.001$ \\
\hline RELIABILITY & $3.57 \pm 0.97$ & $2.45 \pm 0.76$ & 0.002 \\
\hline
\end{tabular}

Table VIII. Upload centre differences according to scores

\begin{tabular}{lccc} 
Variables & $\beta$-coefficient & Confidence interval & $P$-value \\
HONcode & 0.217 & $-0.499,0.933$ & 0.539 \\
\hline RELIABILITY score & 0.434 & $-0.933,1.801$ & 0.521 \\
\hline Video dration & 1.054 & $-2.058,4.167$ & 0.493 \\
\hline Like & 0.008 & $-0.006,0.022$ & 0.256 \\
\hline Dislike & -0.071 & $-0.212,0.069$ & 0.305 \\
\hline Upload centre: & & & \\
\hline 2-1 & 1.490 & $-0.315,3.297$ & 0.102 \\
\hline $3-1$ & 2.165 & $-0.552,4.883$ & 0.114 \\
\hline $4-1$ & 3.960 & $1.389,6.530$ & 0.004 \\
\hline
\end{tabular}

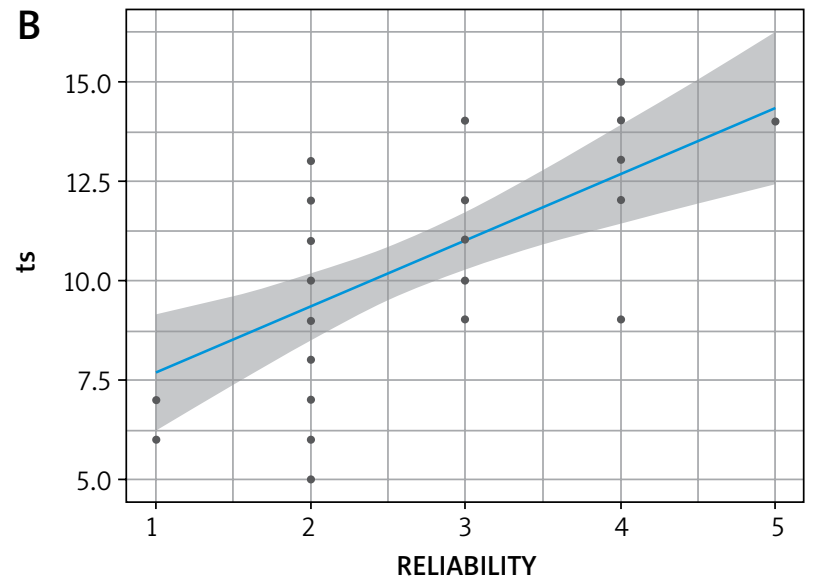

Figure 1. Pericardiocentesis checklist scores of videos and changes according to HONcode, RELIABILITY, and video duration shown in scatterplot

tients is that they should prefer university hospitals or educational hospital posts in the selection of informative and educational videos. As we have shown in our study, it has been shown in other studies that videos made by professionals of educational institutions are more reliable $[13,14]$.

In some studies, evaluating YouTube videos in terms of education, it has been observed that YouTube is beneficial for learning and the videos are sufficient $[15,16]$. Also, it has been stated in most publications that the video quality is not sufficient $[17,18]$. It is noteworthy that publications which include sufficient video quality are containing short and specific procedures videos [15]. It is known that YouTube videos are the most frequently used visual education resource in surgical education, so it is important for users to evaluate videos in terms of reliability by using procedures as well as evaluating scientific publications $[19,20]$.

There were few references of sharing in the videos. This finding is consistent with previous studies, which have found that online information can offer an unbalanced view with little or poor referencing to scientific data [21]. Evidence in the literature proves that multimedia-oriented learning has advantages in terms of understanding complex temporal and spatial events [22].

With this study, we showed that the training videos uploaded to YouTube are not always educational at the desired level. The lack of training videos on highly reliable 
cardiology and cardiothoracic surgeons' websites can lead novices to use videos on YouTube for educational purposes. For this reason, it may be useful to check the educational suitability of videos using special tools such as HONcode, RELIABILITY, and global quality score to ensure that these trainees develop satisfactorily.

There are limitations of the study. First, the lack of a specific YouTube scoring utility and scoring system. After defining the steps of pericardiocentesis according to a reference consensus reports, we tried to overcome this limitation with specific tools such as the global quality score, HONcode, Modified Discern, and JAMA. Scoring systems such DISCERN, HONCODE, etc. are quite general or are geared more towards patients than proceduralists. Other video channels such as Dailymotion were not considered. Our study was snapshot searching of YouTube videos, because of this after study period finished videos might be increased or revised by uploaders.

\section{Conclusions}

YouTube contains variable quality videos related to pericardiocentesis. The educational value of these videos up until now is questionable. It might be recommended that healthcare trainers be aware of and adopt the developing technology, and they might make prefer videos uploaded from university hospitals and educational hospitals.

\section{Disclosure}

Authors report no conflict of interest.

\section{References}

1. Krikorian JG, Mancock EW. Pericardiocentesis. Am J Med 1978; 65: 808-814.

2. Ristic AD, Imazio $M$, Adler $Y$, Anastasakis $A$, Badano LP, Brucato $A$, Caforio ALP, Dubourg O, Elliott P, Gimeno J, Helio T, Klingel K, Linhart A, Maisch B, Mayosi B, Mogensen J, Pinto Y, Seggewiss H, Seferović PM, Tavazzi L, Tomkowski W, Charron P. Triage strategy for urgent management of cardiac tamponade: a position statement of the European Society of Cardiology Working Group on Myocardial and Pericardial Diseases. Eur Heart J 2014; 35: 2279-2284.

3. Velasco E, Agheneza T, Denecke K, Kirchner G, Eckmanns T. Social media and internet-based data in global systems for public health surveillance: a systematic review. Milbank Q 2014; 92: 7-33.

4. Morahan-Martin JM. How internet users find, evaluate, and use online health information: a cross-cultural review. Cyberpsychol Behav 2004; 7: 497-510.
5. YouTube. YouTube Press. https://www.YouTube.com/yt/about/press/ (2020, accesed 12.3.2020)

6. Laversin S, Baujard V, Gaudinat A, Simonet MA, Boyer C. Improving the transparency of health information found on the Internet through the HONcode: a comparative study. Stud Health Technol Inform 2011; 169: 654-658.

7. Charnock D, Shepperd S, Needham G, Gann R. DISCERN: an instrument for judging the quality of written consumer health information on treatment choices. J Epidemiol Community Health 1999; 53: 105-111.

8. Bernard A, Langille M, Hughes S, Rose C, Leddin D, van Zanten SV. A systematic review of patient inflammatory bowel disease information resources on the World Wide Web. Am J Gastroenterol 2007; 102: 2070-2077.

9. Kaicker J, Dang W, Mondal T. Assessing the quality and reliability of health information on ERCP Using the DISCERN Instrument. Health Care Current Reviews 2013; 1: 104.

10. Tsang TS, Seward JB. Pericardiocentesis under echocardiographic guidance. Eur J Echocardiogr 2001; 1: 68-9.

11. Maggiolini S, Gentile G, Farina A, De Carlini CC, Lenatti L, Meles E, Achilli F, Tempesta A, Brucato A, Imazio M. Safety, efficacy and complications of pericardiocentesis by real-time echo-monitored procedure. Am J Cardiol 2016; 117: 1369-1374.

12. Deal SB, Alseidi AA. Concerns of quality and safety in public domain surgical education videos: an assessment of the critical view of safety in frequently used laparoscopic cholecystectomy videos. J Am Coll Surg 2017; 225: 725-730.

13. Lee JS, Seo HS, Hong TH. YouTube as a source of patient information on gallstone disease. World J Gastroenterol 2014; 20: 4066-4070.

14. Madathil KC, Rivera-Rodriguez AJ, Greenstein JS, Gramopadhye AK. Healthcare information on YouTube: a systematic review. Health Informatics J 2015; 21: 173-194.

15. McKee HD, Jhanji V. Learning DMEK from YouTube. Cornea 2017; 36: 14771479.

16. Ruiz-Roca JA, Martínez-Izquierdo A, Mengual-Pujante D, Pons-Fuster López E, López-Jornet P. Is YouTube a useful tool for oral care in patients with Parkinson's disease? Spec Care Dentist 2020; 40: 464-469.

17. Keskinkılıç Yağız B, Yalaza M, Sapmaz A. Is YouTube a potential training source for total extraperitoneal laparoscopic inguinal hernia repair? Surg Endosc 2020 May 4. doi: 10.1007/s00464-020-07596-3. Epub ahead of print.

18. de'Angelis N, Gavriilidis P, Martínez-Pérez A, Genova P, Notarnicola M, Reitano E, Petrucciani N,Abdalla S, Memeo R, Brunetti F, Clotilde Carra M, Di Saverio S, Celentano V. Educational value of surgical videos on YouTube: quality assessment of laparoscopic appendectomy videos by senior surgeons vs. novice trainees. World J Emerg Surg 2019; 9: 14-22.

19. Rapp AK, Healy MG, Charlton ME, Keith JN, Rosenbaum ME, Kapadia MR. YouTube is the most frequently used educational video source for surgical preparation. J Surg Educ 2016; 73: 1072-1076.

20. Mota P, Carvalho N, Carvalho-Dias E, Costa MJ, Correia-Pinto J, Lima E. Videobased surgical learning: improving trainee education and preparation for surgery. J Surg Educ 2018; 75: 828-835.

21. Cline RJW, Haynes KM. Consumer health information seeking on the Internet: the state of the art. Health Educ Res 2001; 16: 671-692.

22. Pitcher GS, Newton DH, Amendola MF. Common femoral artery access on youtube: what practices are being shown and who is delivering the message? J Surg Educ 2017; 74: 455-458. 